\title{
The CALICE hadron calorimeters - test beam results
}

\author{
Jaroslav Cvach $^{1+}$ \\ Institute of Physics of the ASCR, v.v.i. \\ Na Slovance 2, 18221 Prague 8, Czech Republic \\ E-mail: cvach@fzu.cz
}

We present concepts and beam test results of highly granular calorimeter prototypes optimized for the application of particle flow algorithms. A scintillator-steel hadron calorimeter using SiPMs as photodetectors (AHCAL) has been tested in electron and hadron beams at CERN and Fermilab in the energy range $1-80 \mathrm{GeV}$. More than $7600 \mathrm{SiPMs}$ - the highest number ever used - performed well over the period longer than 2 years and did not show an increase of noise. The analysis of the first part of data from hadron beams leads to the energy resolution of $61 \% / \sqrt{ } E$ which can be further improved to $49 \% / \sqrt{ } E$ applying energy dependent weights. The data on the longitudinal and transverse shower shapes allow discriminate among hadronization models of GEANT4. Specifically QGSP_BERT and LHEP prediction were compared to the data. Further we present the concept of a Digital Hadron Calorimeter (DHCAL) with two lines of R\&D following different read-out and integration approach. Both are based on glass resistive pad chambers with $1 \mathrm{~cm}^{2}$ pad read-out, alternative amplification techniques like GEMs or MICROMEGAS are also being considered. One series of studies applies a single threshold (1bit) to the signal charges, providing digital readout with the front end part integrated on the pad board. We report on the measurements with a small scale prototype in the Fermilab test beam using muons, positrons, pions, and protons and in the laboratory using cosmic rays. An alternative approach is to use semi-digital readout electronics following a design close to the ILD detector concept. The electronics will not require active cooling and rely on power-pulsing. A small prototype was built and tested with success at the CERN PS test beam in 2008. A few GRPCs as large as $1 \mathrm{~m}^{2}$ were built with emphasis on minimized dead zones and optimized gas flow. The GRPCs were tested with an electronics board of the same size, containing $14464-$ channel ASICs, representing the largest ever built chamber with embedded electronics. The results will serve as the basis for the design of the fully instrumented $1 \mathrm{~m}^{3}$ CALICE test beam digital calorimeter.

European Physical Society Europhysics Conference on High Energy Physics EPS-HEP 2009, Krakow , Poland July 16-22, 2009

$\begin{array}{ll}1 & \text { Speaker } \\ + & \text { representing the CALICE Collaboration }\end{array}$




\section{Introduction}

The CALICE collaboration develops highly granular calorimeters for detectors at the ILC optimized for particle flow algorithm (PFLOW) [1]. The calorimeter prototypes have been extensively tested in particle beams at DESY, CERN and Fermilab. The results of the electromagnetic calorimeters (ECAL) are reviewed at this conference in [2]. In this contribution we concentrate on concepts and test beam results of highly granular hadron calorimeters. We distinguish between two main groups: analogue calorimeter (AHCAL) with the cell granularity of $\sim 10 \mathrm{~cm}^{2}$ and amplitude read-out with standard ADCs and digital/semi-digital calorimeters (DHCAL) with cell granularity $\sim 1 \mathrm{~cm}^{2}$ and amplitude read-out with $1 /(2-3)$ bits.

\section{The analog hadron calorimeter AHCAL}

The AHCAL consists of small plastic scintillator tiles $0.5 \mathrm{~cm}$ thick with individual readout by silicon photomultipliers (SiPMs) [3] arranged in layers between $2 \mathrm{~cm}$ thick stainless steel absorber plates. The size of the scintillator tiles ranges from $3 \times 3 \mathrm{~cm}^{2}$ in the center to $12 \times 12$ $\mathrm{cm}^{2}$ on the outer edge of the $1 \mathrm{~m}^{2}$ detection plane. In total, the hadron calorimeter volume of 1 $\mathrm{m}^{3}$ has 38 sensitive layers, amounting to a depth of $4.5 \lambda_{I}$, with a total of 7608 scintillator cells. The scintillator-steel tail catcher and muon tracker (TCMT) with total depths of $5.8 \lambda_{I}$ stands behind the AHCAL. In front of the AHCAL, SiW ECAL [2] was mounted with a depth of 24 radiation lengths $X_{0}$ or $1 \lambda_{I}$.

\subsection{Detector performance and calibration}

The detector was operated in the test beam during years 2006-9. In this contribution we report results from the CERN beam H6 in the year 2007. The calibration of the SiPM photodectors and the whole detector was performed using a built-in LED calibration system [4], dedicated runs with muon beam at the beginning of the data taking campaign and isolated track segments in hadronic showers throughout the whole period [5]. The pedestal distribution of signals from all SiPMs was permanently monitored. Approximately $2 \%$ channels were dead and only eight SiPMs showed increase of pedestal with time. This demonstrates long-term stable behaviour of the AHCAL.

Data with pion beam in the energy range $2-80 \mathrm{GeV}$ were reconstructed using the program chain MARLIN. The correction on the SiPM nonlinearity was applied based on test bench measurement of each SiPM at ITEP Moscow and time stability of the corection monitored by the LED system. The linearity and energy resolution are shown in Fig. 1. as a function of beam energy as black points. The AHCAL is slightly non-compensating calorimeter with $\mathrm{e} / \pi \sim 1.15$. High granularity of the calorimeter allows to identify clusters of higher energy density deposits corresponding to $\pi^{0}$ decays. Assigning them a lower weight we showed (red points) [5] that both linearity and energy resolution can be improved (the stochastic term decreases by $20 \%$ to the value $49.2 \% / \sqrt{E}$ ). The energy resolution is shown for the complete CALICE setup 
$(\mathrm{ECAL}+\mathrm{AHCAL}+\mathrm{TCMT})$. The spread of reconstructed energy and resolution for multiple measurements at the same energy is due to the missing temperature correction
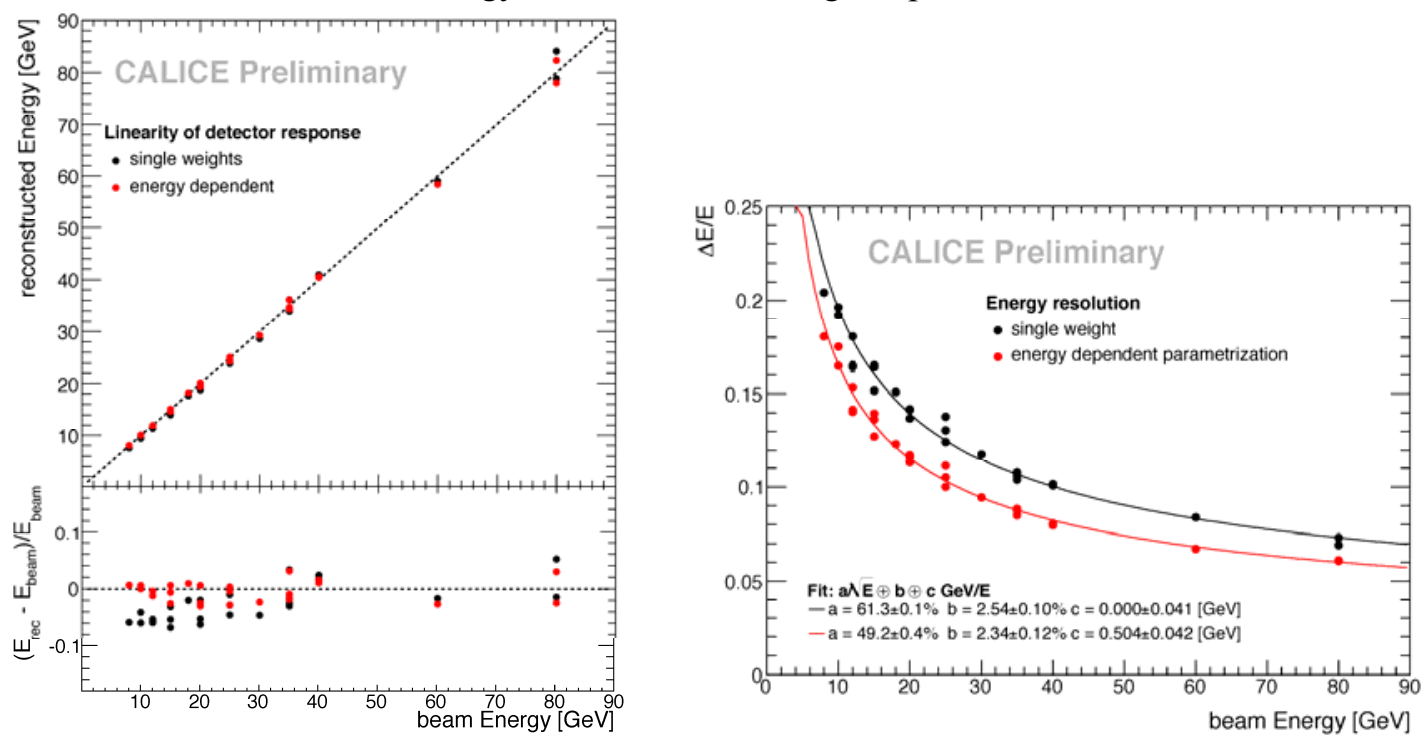

Figure 1 (left) Reconstructed energy of the complete CALICE setup for hadrons as a function of beam energy without (black) and with (red) energy weighting. The lower panel shows the relative deviation of the reconstructed energy from the beam energy (right) Energy resolution of the complete CALICE setup as a function of beam energy without (black)/ with (red) energy weighting.

\subsection{Properties of hadronic showers in AHCAL}

Thanks to the high longitudinal and lateral segmentation of the calorimeter we have started a detail comparison of hadronic shower properties with different hadronization models in GEANT4. We selected two different approaches in the description of the shower development: LHEP and QGSP_BERT [6].
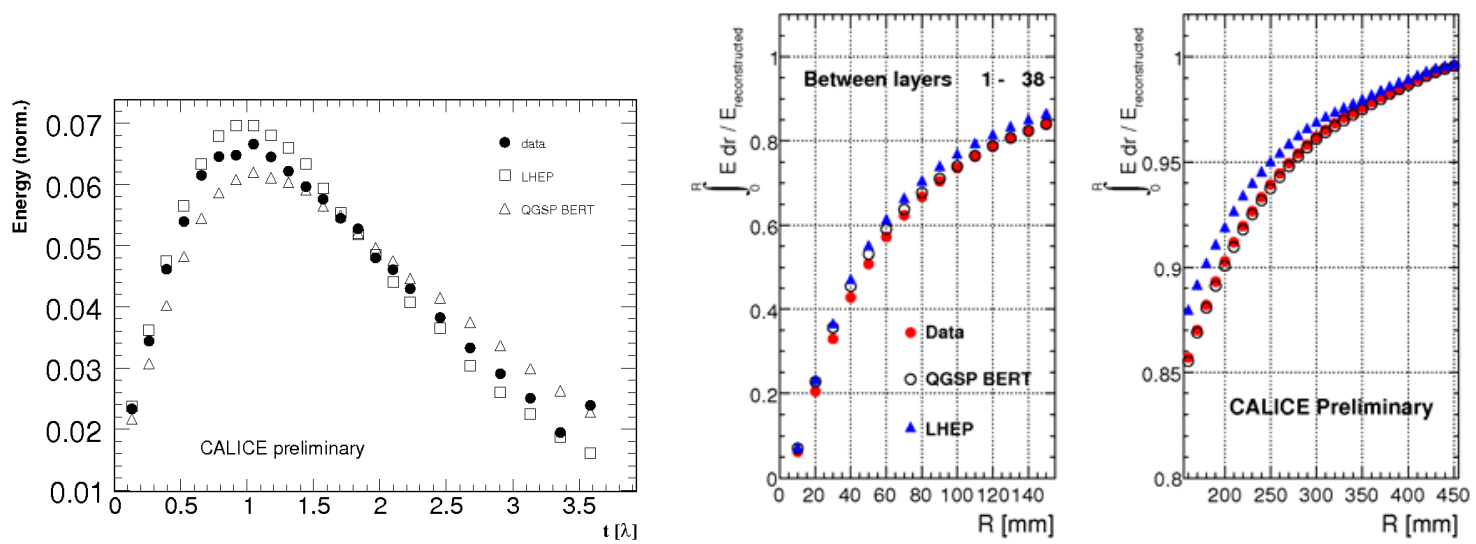

Figure 2 (left) Longitudinal shower profile of $10 \mathrm{GeV}$ pions for data and GEANT4 models. (center and right) Lateral shower containment of $18 \mathrm{GeV}$ pions.

In Fig. 2 left, the longitudinal profile for $10 \mathrm{GeV}$ pion showers is plotted as a dependence of shower energy density on the distance $t$ from the AHCAL front face. The LHEP showers are narrower and QGSP_BERT wider than the data. The lateral shower profile was studied in form 
of energy density distribution $\rho_{E}$ as a function of the radial coordinate $R^{1}$. The lateral profile exhibits a narrow core, representing the electromagnetic component from $\pi^{0}$, s produced in the shower development, and a halo caused by hadron production. After integration over all AHCAL layers the lateral profile is investigated as a function of $R$ or via the cumulative integral from the lowest bin up to the $R$-bin, and then normalized to the total reconstructed energy. The comparison with Monte Carlo models is shown in Fig. 2 centre (right) for the core (tail) of the shower. QGSP_BERT appears to better reproduce the fractional energy distribution in the shower tail, LHEP showers are narrower than $18 \mathrm{GeV}$ pion data.

\section{Digital hadron calorimeters DHCALs}

Digital calorimeters aim at higher granularity than analogue calorimeters at the expense of reduced energy information from a cell. The higher granularity will reduce the confusion between charged and neutral particles but increases significantly number of electronic channels. From the point of view of the detector cost and high detection sensitivity, gas hadronic calorimeters represent the best choice. Besides the glass resistive plate chambers (GRPC) the other two possibilities investigated for use in the ILC calorimetry are MICROMEGAS using bulk technology and GEMs $[7,8]$. The chambers of the area of $\sim 10^{3} \mathrm{~cm}^{2}$ were tested in the beam and serve as a test ground for construction of large $1 \mathrm{~m}^{2}$ chambers.

\subsection{Digital and semi-digital GRPC calorimeters}

The GRPC is made of two thin glass plates separated by spacers. High voltage is applied over $1.2 \mathrm{~mm}$ gas gap trough a thin layer of resistive painting on the outer side of the plate. The electronic board separated by Mylar contains read-out pads of $1 \mathrm{~cm}^{2}$. The operation voltage for the avalanche mode in the range $6300-7400 \mathrm{~V}$ depends on the gas mixture. In calorimeter the GRPCs are inserted between $2 \mathrm{~cm}$ thick steel absorber plates. Signals from pads are read by a thin 64 channel ASIC which is embedded with electronics inside the calorimeter. Depending on the number of thresholds in the signal read-out we distinguish between digital (1) and semidigital (2-3) calorimeters. The prototypes of both types of calorimeters were in test beam in 2008-9.

The digital calorimeter made of $20 \times 20 \mathrm{~cm}^{2}$ RPCs was exposed to $120 \mathrm{GeV}$ muon, 120 $\mathrm{GeV}$ proton and $1-16 \mathrm{GeV}$ pion/positron beams at Fermilab. Fig. 3 left shows the sum of hits in the six layers $\left(1.2 X_{0}, 1536\right.$ channels) for electromagnetic showers from the positron beam. The red histograms correspond to a simulation by a GEANT4 based program with a model of RPC response. It is in a reasonable agreement with the data (dots). Both the measured and simulated data are adequately described by a Gaussian [9].

The semi-digital calorimeter consists of 5 chambers $33 \times 8 \mathrm{~cm}^{2}$. It uses a new ASIC (HARDROC) which deals with 64 channels and is connected via daisy chain. The calorimeter was exposed to the CERN PS beam of $1-6 \mathrm{GeV}$ and the data allowed study the development of the first phase of hadronic showers. Based on the experience with the small prototype a large

\footnotetext{
$R_{i}=\left(\left(x_{i}-x_{\text {track }}\right) 2+\left(y_{i}-y_{\text {track }}\right)^{2}\right)^{1 / 2}, x_{i}\left(y_{i}\right), x_{\text {track }}\left(y_{\text {track }}\right)$ are the $x(y)$ coordinates of the scintillator tile and of the track impact point at the tile layer. The shower energy density, defined as the mean energy sum per event per unit ring area, is then measured event by event in bins of the radial coordinate $R$.
} 
$1 \mathrm{~m}^{2}$ GRPC was tested this summer at CERN hadron beam [10]. It is the largest chamber built with such granularity.
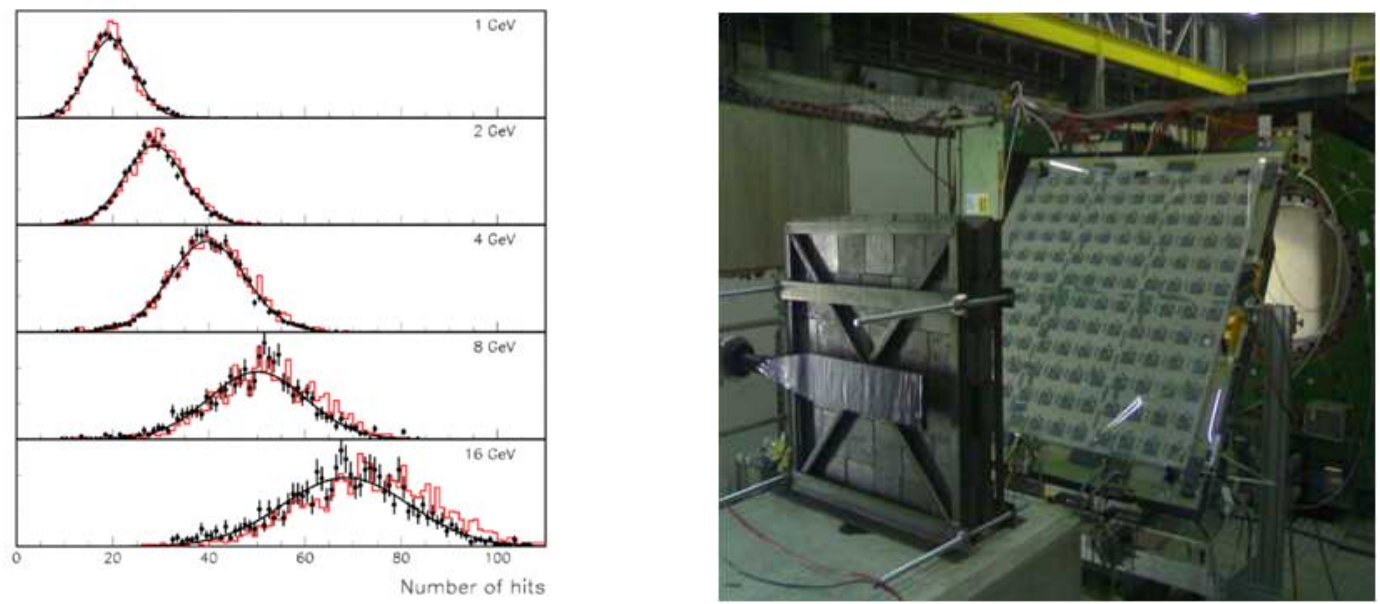

Figure 3 (left) Normalized distribution of the sum of hits in the six layer DHCAL (points), GEANT4 simulation (red histogram) and Gaussian fit (full line). (right) The view of a $1 \mathrm{~m}^{2}$ GRPC behind a 1 $\lambda_{I}$ wall at the CERN test beam.

Acknowledgment. I would like to thank to all my colleagues from the CALICE Collaboration who contributed to the results presented in this review. Especially I thank to J. Blaha, E. Garutti, I. Laktinekh, J. Repond and D. Ward for many useful discussions. This work was supported by the Ministry of Education of the Czech Republic under the projects LC527 and INGO-LA09042 and by the Commission of the EC under the $6^{\text {th }}$ Framework Programme, contract number RII3026126.

\section{References}

[1] M. A. Thomson, Particle Flow Calorimetry and the PandoraPFA Algorithm, [arXiv:0907.3577v1].

[2] C. Cârloganu, Electromagnetic Calorimetry for ILC, these proceedings.

[3] G. Bondarenko et al., NIM A 442 (2000) 187.

[4] I. Polák, Development of the AHCAL calibration system, ECFA and GDE meeting, Valencia 2006.

[5] F. Simon, Hadronic showers in the CALICE calorimeter prototypes, in proceedings of TIPP09.

[6] J. Apostolakis et al., Hadronic Shower Shape Studies in GEANT4, CERN-LCGAPP-2007-02.

[7] C. Adloff et al., MICROMEGAS chambers for hadronic calorimetry at a future linear collider, submitted to JINST.

[8] J. Yu, Development of GEM Based Digital Hadron Calorimeter, in proceedings of LCWS/ILC 2007 $567-572$.

[9] B. Bilki et al., Measurement of positron showers with a digital hadron calorimeter, 2009 JINST 4 P04006; Measurement of the rate capability of RPC, 2009 JINST 4 P06003; Calibration of a digital hadron calorimeter with muons, 2008 JINST 3 P05001.

[10] I. Laktineh, Semi-digital Hadronic Calorimeter for Future High Energy Physics Experiments, Journal of Physics: Conference Series 160 (2009) 012030. 